\title{
Aircraft vertical profiles during summertime regional and Saharan dust scenarios over the north-western Mediterranean Basin: aerosol optical and physical properties.
}

Jesús Yus-Díez ${ }^{1,2}$, Marina Ealo ${ }^{1,2}$, Marco Pandolfi ${ }^{1}$, Noemí Perez ${ }^{1}$, Gloria Titos $^{1,3}$, Griša Močnik ${ }^{4,5}$, Xavier Querol ${ }^{1}$ and Andrés Alastuey ${ }^{1}$

${ }^{1}$ Institute of Environmental Assessment and Water Research (IDAEA-CSIC), C/Jordi Girona 18-26, 08034, Barcelona, Spain.

${ }^{2}$ Departament de Física Aplicada - Meteorologia, Universitat de Barcelona, C/Martí i Franquès, 1., 08028, Barcelona, Spain ${ }^{3}$ Andalusian Institute for Earth System Research (IISTA-CEAMA), University of Granada, Autonomous Government of Andalusia, Granada, Spain.

${ }^{4}$ Slovenia Center for Atmospheric Research, University of Nova Gorica, Vipavska 11c, SI-5270 Ajdovščina, Slovenia.

${ }^{5}$ Department of Condensed Matter Physics, Jozef Stefan Institute, Jamova 39, SI-1000 Ljubljana, Slovenia.

Correspondence: jesus.yus@idaea.csic.es 


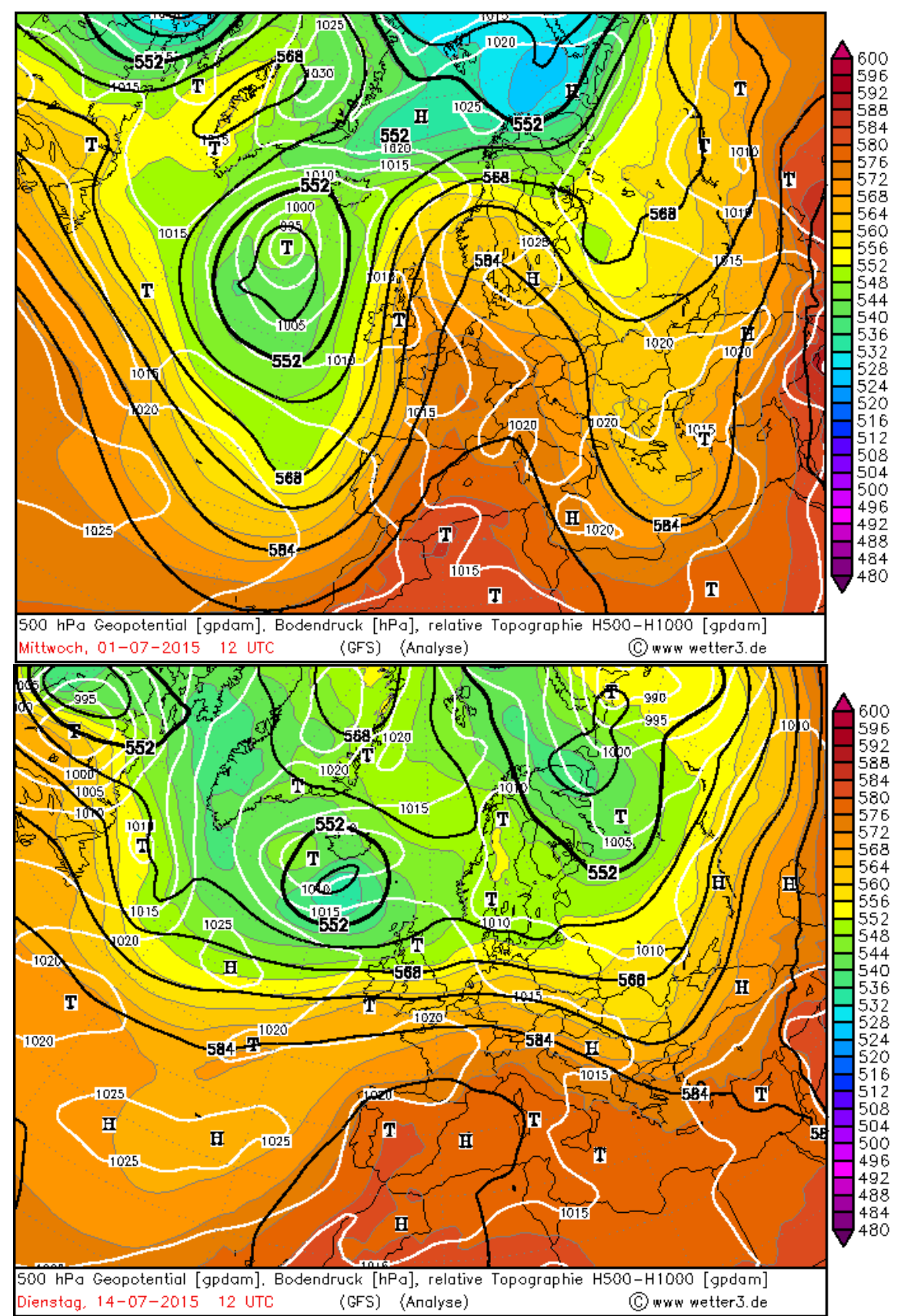

Figure S1: Synoptic mete orology with the $500 \mathrm{hPa}$ Geopotential and surface pressure at 12:00 UTC for the 7th and 16th of July of 2015 as modelled by the reanalysis model ERA-INTERIM, (C) www.wetter3.de. 

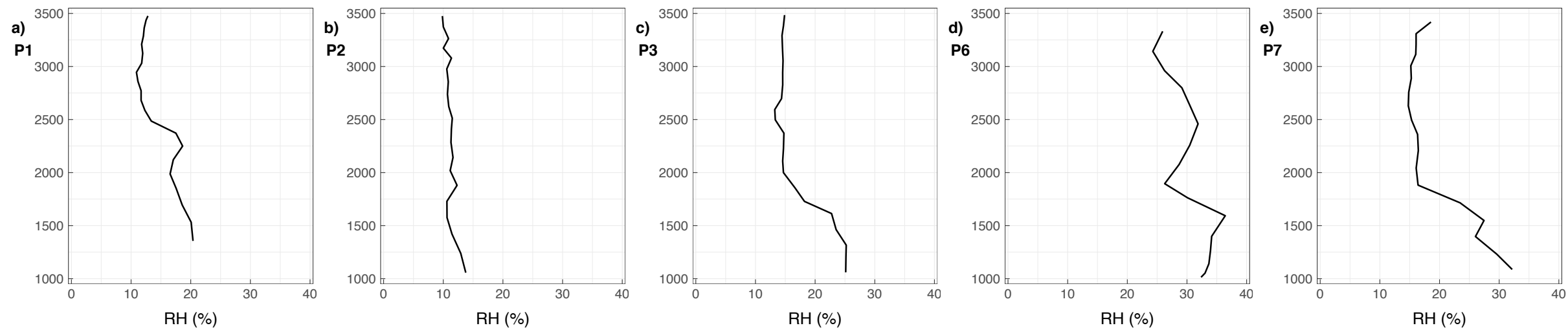

Figure S2: Vertical profiles of relative humidity (RH (\%)) for a) P1, b) P2, c) P3, d) P6, and e) P7 as measured by the Kestrel unit placed in the aircraft.
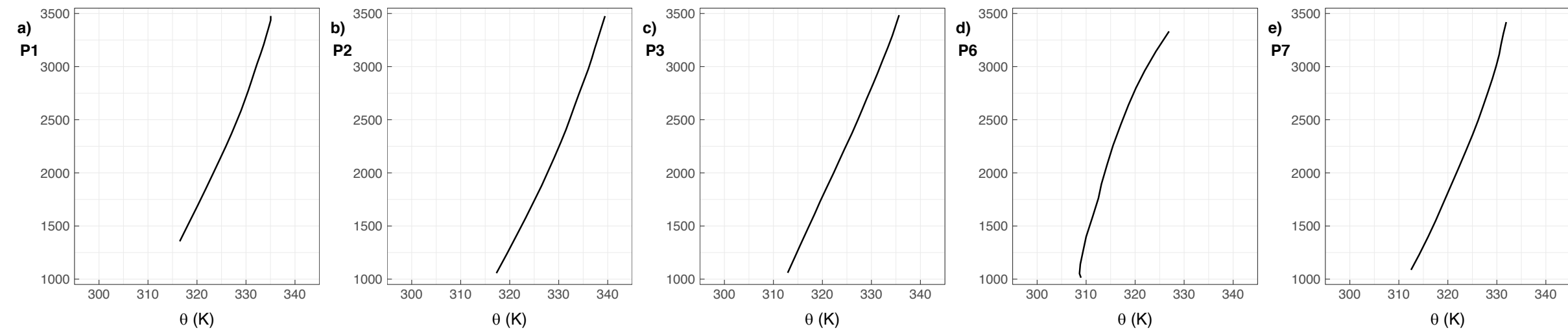

Figure S3: Vertical profiles of potential temperature $(\theta(K))$ for a) P1, b) P2, c) P3, d) P6, and e) P7 as measured by the Kestrel unit placed in the aircraft. 


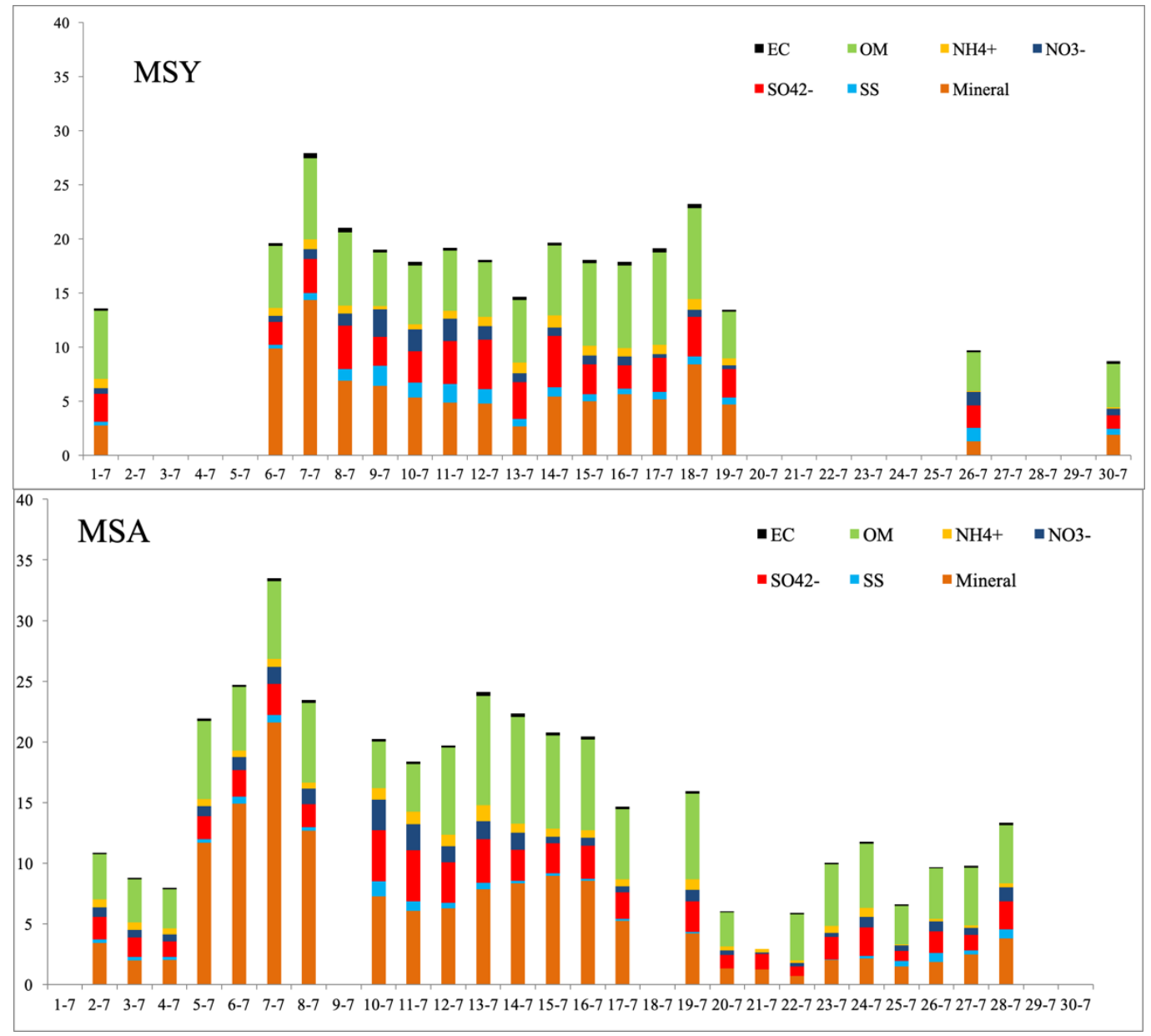

Figure S4: Daily off-line filter base chemistry analysis during the instrumented flights period of July 2015 for MSA (upper panel) and MSY (lower panel). 
Table S1: Comparison between aircraft borne measurements for P1 versus the in-situ measurements of MSA station as a reference at the same time that the flight was at a height of $1500 \mathrm{~m}$ a.s.1. approximately.

\begin{tabular}{|c|c|c|c|c|c|c|c|c|c|c|c|}
\hline & $P M_{10}\left(\mu g m^{-3}\right)$ & $P M_{2.5}\left(\mu g m^{-3}\right)$ & $P M_{1}\left(\mu g m^{-3}\right)$ & $P M_{1 / 10}$ & $P M_{1 / 2.5}$ & $\sigma_{s p 525 n m}\left(M^{-1}\right)$ & $\sigma_{a p} 590 \mathrm{~nm}\left(\mathrm{Mm}^{-1}\right)$ & SAE & AAE & $\mathbf{G}$ & SSA \\
\hline P1 - FLIGHT & 20.3 & 18.38 & 13.38 & 0.66 & 0.73 & 37.85 & 2.59 & 0.93 & 1.76 & 0.59 & 0.87 \\
\hline P1 - MSA IN-SITU & 38.4 & 20.8 & 14.2 & 0.37 & 0.68 & 41.44 & 3.06 & 0.90 & 2.12 & 0.62 & 0.86 \\
\hline DIFF (\%) & 47.1 & 11.6 & 5.8 & -78.2 & -6.6 & 8.7 & 15.36 & -4.5 & 16.8 & 4.55 & -0.22 \\
\hline
\end{tabular}

